\title{
Harmonic Output of the Synchronous Rectifier
}

\author{
Paul Selgin
}

\begin{abstract}
If a sine-wave current or voltage of generic frequency $f$ is admitted into a switching device that inverts its polarity with frequency $F$, the output will contain all the positive frequencies $\pm f+N F$, with $N$ an odd integer. This fact, as well as the amplitude and phase of each component, results from the expansion of the output in a complex Fourier series. Application to particular cases illustrates the behavior of the synchronous rectifier in cases when the input contains nonsynchronous components. In particular, the selectivity and damping associated with the synchronous rectifier are made the object of comparative considerations.
\end{abstract}

\section{Use of the Synchronous Rectifier ${ }^{1}$}

Rectification based on the unilateral conductivity of semi conductors, of gas or vapor discharges, and of vacuum tubes, is a comparatively recent development. Rectification based on mechanical switching, timed to coincide with the inversion in polarity, is much older: its first example was the two-pole commutator used in the early permanent magnet dynamos. Mechanically operated synchronous rectifiers present a problem when they are required to handle considerable power because of the difficulty in mechanically commutating large currents, although there are ways of overcoming this by interrupting the circuit at the instants when the current is at a minimum; so-called "contact rectifiers", studied and used chiefly in Germany, are based on this principle.

In the measurement field ${ }^{2}$ synchronous rectifiers are useful chiefly in combination with "choppers", to permit the use of a-c amplifiers to handle a d-c signal. Ordinary unidirectional rectifiers, such as diodes, do not discriminate between the desired signal and noise, and the only way to reduce the noise present in the output is to insert a selective band-pass filter somewhere in the system. The output of synchronous rectifiers, on the other hand, contains d-c only when the input contains the synchronous frequency itself or an odd multiple thereof. Therefore only discrete infinitesimal portions of the noise spectrum will contribute to the d-c level, and because of the random phase the net total of an infinite number of these infinitesimal contributions will be zero. In other words, the synchronous rectifier d-c output is unaffected by noise. While this is a very desirable feature, it does not eliminate the need for filtering; a-c components of the output have to be reduced to prevent them from masking the $d$-c component. This is easier to achieve than the selective transmission of a narrow band; however, if the a-c components are cut down below a certain point the system becomes very sluggish and slow to respond to changes in the d-c level. Basically, the difficulty is the same whatever the system used; it is impossible

1 "Synchronous rectifiers", as discussed here, include types wherein the rectification is due to mechanical interruption of a circuit at the synchronizing frequency. The analysis applies only approximately to synchronous detectors, where a synchronizing voltage is applied, together with the signal voltage, to where a synchronizing voltage is applied, togeth

suitable points of a diode or vacuum tube circuit.
2 Walter, Zeitschrift $\mathrm{f}$. Tech. Physik 13,363 and 436 (1932). Morton, Trans. 2 Walter, Zeitschrift $\mathrm{f}$. Tech. Physik 13, 363 and 436 (1932). Morton, Trans.
Faraday Soc. 46,818 (1934); Astbury, J. Sci. Inst. 17, 25 (1940). Michaels and Curtis, Rev. Sci. Instr. 12, 444 (Sept. 1941). to eliminate noise entirely without sacrificing the ability to follow arbitrary variations in d-c level.

Aside from the specific application mentioned above, it is of some interest to investigate the behavior of the synchronous rectifier generally, taking as the starting point the Fourier analysis of the output when a sine-wave input of arbitrary frequency phase and amplitude, is impressed upon the rectifier, which is assumed to do nothing more than invert periodically the polarity of the input at a given fixed frequency $F$.

\section{Initial Steps in the Fourier Analysis}

Consider a harmonic time function whose phase is changed by $\pi$ at uniform time intervals. The phase inversion may be regarded as the effect of multiplication of the harmonic function by a "square wave" function of value \pm 1 , the sign changing periodically. Let $f$ be the frequency of the harmonic function, hereafter called the "input", and $F$ that of the square wave; let $F$ be designated as the "synchronous frequency".

In general, $f$ and $F$ will be incommensurable. We will suppose, however, that their ratio is rational. This does not, in effect, limit the scope of the analysis, since it will appear that the components of the output approach a limit as the input frequency approaches any arbitrary value through a sequence of rational numbers (with reference to the synchronous frquency taken as unity).

We can therefore write

$$
\frac{f}{F}=\frac{m}{M},
$$

and, in setting up the Fourier analysis, we can ignore the actual frequencies in the interest of simplicity in writing, and use instead the two numbers $m$ and $M$, assuming that within the time interval $2 \pi$ the input function goes through $m$ complete periods, and the square wave function through $M$ periods. The situation is illustrated in figure 1 . We can see that the output will be periodic, of period $2 \pi$. This time interval is, in fact, the least common multiple of both the input and synchronous periods.

Our substitutions enable us to write the input function in the form: 
$\nu_{i}(t)=V_{i} \cos (m t+\Phi)=a \cos m t+b \sin m t$

$$
\left[V_{i}=\sqrt{a^{2}+b^{2}} ; \tan \phi=\frac{b}{a}\right]
$$

and the output in the form of a complex Fourier series: ${ }^{3}$

$$
\nu_{0}(t)=\sum_{n=-\infty}^{\infty} C_{n} e^{-i n t},
$$

which is equivalent to the more familiar, but less convenient in this case, Fourier series with real coefficients:

$$
\nu_{0}(t)=\sum_{n=0}^{\infty}\left(a_{n} \cos n t+b_{n} \sin n t\right),
$$

provided the complex coefficients $C_{n}$ in (3) and the real coefficients $a_{n}$ and $b_{n}$ in (4) are mutually bound by the equations

$$
\left.\begin{array}{c}
C_{n}=\frac{1}{2}\left(a_{n}-i b_{n}\right) \\
C_{-n}=\frac{1}{2}\left(a_{n}+i b_{n}\right)
\end{array}\right\}
$$

The square-wave synchronous function of figure 1 may be designated provisionally by $S(t)$. The output may, therefore, be written also in the form of a factor:

$$
\nu_{0}(t)=S(t) v_{i}(t)=S(t)(a \cos m t+b \sin m t),
$$

$$
\begin{aligned}
\int_{-\pi}^{\pi} S(t) e^{i(m-n) t} d t= & \int_{-\pi}^{-\pi+\frac{\pi}{M}} e^{i(m-n) t} d t-\int_{-\pi+\frac{\pi}{M}}^{-\pi+\frac{2 \pi}{M}} e^{i(m-n) t} d t+\ldots+\int_{\pi-\frac{2 \pi}{M}}^{\pi-\frac{\pi}{M}} e^{i(m-n) t} d t-\int_{\pi-\frac{\pi}{M}}^{\pi} e^{i(m-n) t} d t \\
& =\frac{-i}{m-n}\left\{\left[e^{i(m-n) t}\right]_{-\pi}^{-\pi+\frac{\pi}{M}}-\left[e^{i(m-n) t}\right]_{-\pi+\frac{\pi}{M}}^{-\pi+\frac{2 \pi}{M}}+\ldots+\left[e^{i(m-n) t}\right]_{\pi-\frac{\pi}{M}}^{\pi}-\left[e^{i(m-n) t}\right]_{\pi-\frac{\pi}{M}}^{\pi}\right\} \\
& =\frac{-i}{m-n}\left\{e^{\pi i(m-n) \frac{1-M}{M}}-e^{-\pi i(m-n)}-e^{\pi i(m-n) \frac{2-M}{M}}+e^{\pi i(m-n) \frac{1-M}{M}}+\ldots+e^{\pi i(m-n) \frac{M-1}{M}}-\right. \\
& \left.e^{\pi i(m-n) \frac{M-2}{M}}-e^{\pi i(m-n)}+e^{\pi i(m-n) \frac{M-1}{M}}\right\} \\
= & \frac{-i}{m-n} e^{-\pi i(m-n)}\left\{2 e^{\pi i \frac{m-n}{M}}-2 e^{2 \pi i \frac{m-n}{M}}+2 e^{3 \pi i \frac{m-n}{M}}-\ldots+2 e^{(2 M-1) \pi i \frac{m-n}{M}}-1-e^{2 \pi i(m-n)}\right\}
\end{aligned}
$$

Let us temporarily, for convenience, write:

$$
e^{\pi i \frac{m-n}{M}}=\alpha
$$

and rearrange (9) as follows:

$$
\int_{-\pi}^{\pi} S(t) e^{i(m-n) t}=\frac{2 i}{m-n} \alpha^{-M}\left\{1-\alpha+\alpha^{2}-\alpha^{3}+\alpha^{4}-\ldots-\alpha^{2 M-1}-\frac{1-\alpha^{2 M}}{2}\right\}
$$

? See for example, Churchill, Fourier series and boundary value problems (McGraw-Hill Book Co., Inc., New York, N. Y., 1941). 
Recalling the identity:

$$
1-\alpha+\alpha^{2}-\alpha^{3}+\alpha^{4}-\ldots-\alpha^{2 M-1}=\frac{1-\alpha^{2 M}}{1+\alpha}
$$

equation (9) becomes

$$
\int_{-\pi}^{\pi} S(t) e^{i(m-n) t}=\frac{i}{m-n}\left(\alpha^{-M}-\alpha^{M}\right) \frac{1-\alpha}{1+\alpha}
$$

and restoring the original notation for $\alpha$ :

$$
\int_{-\pi}^{\pi} S(t) e^{i(m-n) t}=\frac{-2 i[\sin \pi(m-n)]\left[\tan \frac{\pi}{2} \frac{m-n}{M}\right]}{m-n}
$$

We can now write an expression for $C_{n}$, the complex Fourier coefficient, from equations (8) and (14): for brevity we will use the notation

$$
\left.\begin{array}{l}
\frac{[\sin \pi(m+n)]\left[\tan \frac{\pi}{2} \frac{m+n}{M}\right]}{\pi(m+n)}=p_{n} \\
\frac{[\sin \pi(m-n)]\left[\tan \frac{\pi}{2} \frac{m-n}{M}\right]}{\pi(m-n)}=q_{n}
\end{array}\right\}
$$

thus obtaining

$$
C_{n}=\frac{i}{2}\left[a\left(p_{n}-q_{n}\right)+i b\left(p_{n}+q_{n}\right)\right] .
$$

\section{Harmonic Spectrum}

The real Fourier coefficients, giving the phase and amplitude of each harmonic in the synchronous rectifier output, may now be written, using eq 16 and the relationships (5). The steps simplify because of the identities:

$$
\left.\begin{array}{l}
p_{-n}=q_{n} \\
q_{-n}=p_{n}
\end{array}\right\}
$$

implicit in eq 15. Using these, and eq 5, we have:

$$
\begin{aligned}
a_{n}=C_{n}+C_{-n} & =\frac{i}{2}\left[a\left(p_{n}-q_{n}+q_{n}-p_{n}\right)\right. \\
\left.+i b\left(p_{n}+q_{n}+q_{n}+p_{n}\right)\right] & \\
=-b\left(p_{n}-q_{n}\right) & \\
b_{n}=i\left(C_{n}-C_{-n}\right) & =-\frac{1}{2}\left[a\left(p_{n}-q_{n}-q_{n}+p_{n}\right)\right. \\
& \left.+i b\left(p_{n}+q_{n}-q_{n}-p_{n}\right)\right] \\
& =-a\left(p_{n}-q_{n}\right),
\end{aligned}
$$

where $a_{n}$ and $b_{n}$ are the Fourier coefficients relative to the $n$th harmonic; the $n$th harmonic, that is, not of the input frequency nor of the synchronous frequency but of that frequency whose period is the least common multiple of the two; or, in other vords, the $n$th harmonic of the frequency at which there is a recurrence of phase coincidence between the input and square-wave functions.

We can readily express the amplitude and phase of this $n$th harmonic: (see eqn 2):

$$
\begin{aligned}
V_{n}=\sqrt{a_{n}^{2}+b_{n}^{2}}=\sqrt{\left(p_{n}^{2}+q_{n}^{2}\right)\left(a^{2}+b^{2}\right)-2 p_{n} q_{n}\left(a^{2}-b^{2}\right)} \\
=V_{i} \sqrt{p_{n}^{2}+q_{n}^{2}-2 p_{n} q_{n} \cos 2 \phi} \\
\quad \tan \phi_{n}=(\cot \phi) \frac{p_{n}-q_{n}}{p_{n}+q_{n}}
\end{aligned}
$$

where $V_{c}$ and $\varphi$ are the amplitude and phase of the input. The analysis of the harmonic spectrum is simplified by the fact that for a given harmonic of order $n$, the coefficients $p_{n}$ and $q_{n}$ do not generally exist together, one or the other being zero except in the particular case when $f$ and $F$, input and synchronous frequencies, are commensurable.

Considering, in fact, eq 15 , we see that, since both $m$ and $n$ are integers by definition, both $p_{n}$ and $q_{n}$ are zero, because of the vanishing of the sines except when $(m+n) / M$ or $(m-n) / M$ are odd integers, in which case the tangents are infinite and $p_{n}$, or $q_{n}$, respectively, takes an interderminate form that has finite nonzero value.

Consequently, $p_{n}$ and $q_{n}$ can coexist only when both $(m+n) / \dot{M}$ and $(m-n) / M$ are odd integers. But if this is true, the sum and difference of these numbers must be even integers, from which we conclude that both ratios $m / M$ and $n / M$ are integers; which means that the input frequency $f$ must be a multiple of the synchronous frequency $F$, which then becomes the fundamental frequency.

This special case will be considered later. Let us first assume that $f$ is not a multiple of $F$. The harmonic spectrum then divides into two families: (a) harmonics corresponding to $(m+n) / M=N$ with $N$ an odd integer; (b) harmonics corresponding to $(m-n) / M=N$ with $N$ an odd integer. In terms of actual frequencies, the frequency of a particular harmonic of order $n$ will be

$$
f_{n}=n \frac{F}{M},
$$

since $F / M$ is the fundamental frequency (fig. 1). Hence the frequencies of family (a):

$$
f_{n}^{\prime}=(N M-m) \frac{F}{M}=-f+N F,
$$

where $N$ must be an odd integer greater than $f / F$.

For the frequencies of family (b) we have, likewise:

$$
f_{n}^{\prime \prime}=f+N F,
$$

where $N$ must be an odd integer greater than $-f / F$.

The grouping of the two families is illustrated in 
figure 2. As for the amplitude and phase values: for family (a) we have

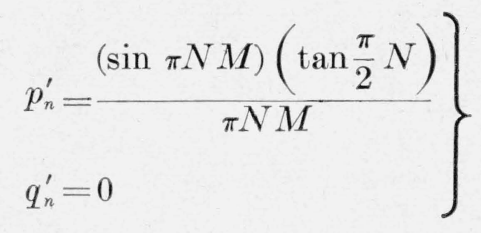

and for family (b):

$$
\begin{aligned}
& p_{n}^{\prime \prime}=0 \\
& \left.q_{n}^{\prime \prime}=\frac{(\sin \pi N M)\left(\tan \frac{\pi}{2} N\right)}{\pi N M}\right\} .
\end{aligned}
$$

The common value of $p_{n}^{\prime}$ and $q_{n}^{\prime \prime}$ is given in indeterminate form and must be evaluated. This can be done by L'Hopital's rule; the result is

$$
p_{n}^{\prime}=\frac{2}{\pi N} \quad q_{n}^{\prime \prime}=\frac{2}{\pi N} .
$$

We can now tabulate the frequency, amplitude and phase values for the two families in the general case when the input and synchronous frequencies are incommensurable:

Frequency: $\left\{\begin{array}{cc}\text { Family (a) } & \text { Family (b) } \\ f_{n}^{\prime}=-f+N F & f_{n}^{\prime \prime}=f+N F \\ \left(N>\frac{f}{F} ; N \text { odd }\right) & \left(N>-\frac{f}{F} ; N \text { odd }\right)\end{array}\right.$

Amplitude: $\quad V_{n}^{\prime}=\frac{2 V i}{\pi N} \quad V_{n}^{\prime \prime \prime}=\frac{2 V i}{\pi N}$

Phase: $\quad \phi_{n}^{\prime}=\frac{\pi}{2}-\phi \quad \phi_{n}^{\prime \prime}=\left\{\begin{array}{l}\phi-\frac{\pi}{2} ;(N<0) \\ \phi+\frac{\pi}{2} ;(N>0)\end{array}\right.$

The following deductions can be drawn from an examination of the data of figure 2 :

1. The amplitude of the harmonics does not depend on the phase of the input.

2. Family (a) may be regarded as a "reflection" of family (b) at the axis of zero frequency, accompanied by a reversal in phase.

3. The two harmonics of greatest amplitude are symmetrically spaced about the frequency $f$ if $f>F$, about $F$ if $f<F$.

4. The conclusions reached may be extended to the case when $f$ is not rational. If, in fact, the input frequency approaches a value $f$ irrationally related to $F$ in such a manner that its subsequent values $f_{1}, f_{2}, f_{3}, \ldots$, are all rationally related to $F$, the $n$th harmonic of the output will take on values $\pm f_{1}+N F, \pm f_{2}+N F, \pm f_{3}+N F, \ldots$ which tend to $\pm f+N F$ as a limit. The restriction imposed

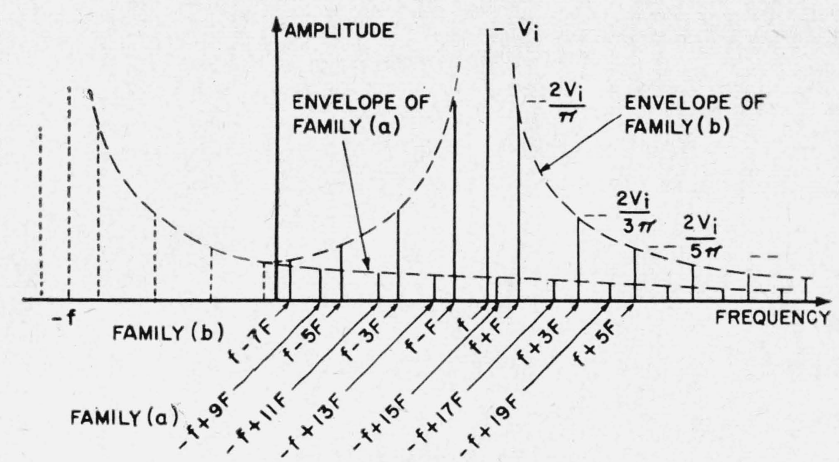

FiguRE 2. Harmonic spectrum in a generic case, showing the presence of two distinct "families".

at the start of the analysis, that $f / F$ be a rational number, may therefora be removed.

5. There is no d-c output in the general case when $f$ is not a multiple of $F$. This can be deduced from figure 2 by inspection. Only frequencies that are odd multiples of the synchronous frequencies contribute to the d-c output.

\section{Commensurable Case}

In the particular case when $f=m F, F$ becomes the fundamental frequency, and $M$ is equal to unity. The expressions for $p_{n}$ and $q_{n}$ (eq 15) then become:

$$
\left.\begin{array}{l}
p_{n}=\frac{[\sin \pi(m+n)]\left[\tan \frac{\pi}{2}(m+n)\right]}{\pi(m+n)} \\
\left.q_{n}=\frac{[\sin \pi(m-n)]\left[\tan \frac{\pi}{2}(m-n)\right]}{\pi(m-n)}\right\}
\end{array}\right\}
$$

and we find that both $p_{n}$ and $q_{n}$ exist for the same values of $n$ : specifically, if $m$ is odd, $p_{n}$ and $q_{n}$ exist together for every even value of $n$; if $m$ is even, for every odd value.

We therefore have only one family of harmonics: those of fraquency:

$$
f_{n}=n F
$$

with the understanding that $n$ can be any positive odd integer when $m=f / F$ is even, and any positive even integer, or zero, when $m$ is odd.

The evaluation of $p_{n}$ and $q_{n}$ can be carried out as before; we obtain:

$$
\left.\begin{array}{l}
p_{n}=\frac{2}{\pi(m+n)} \\
q_{n}=\frac{2}{\pi(m-n)}
\end{array}\right\}
$$

hence the amplitude of the $n$th harmonic (eq 19):

$$
V_{n}=\frac{4 V_{i} \sqrt{m^{2} \sin ^{2} \phi+n^{2} \cos ^{2} \phi}}{\pi\left(m^{2}-n^{2}\right)}
$$



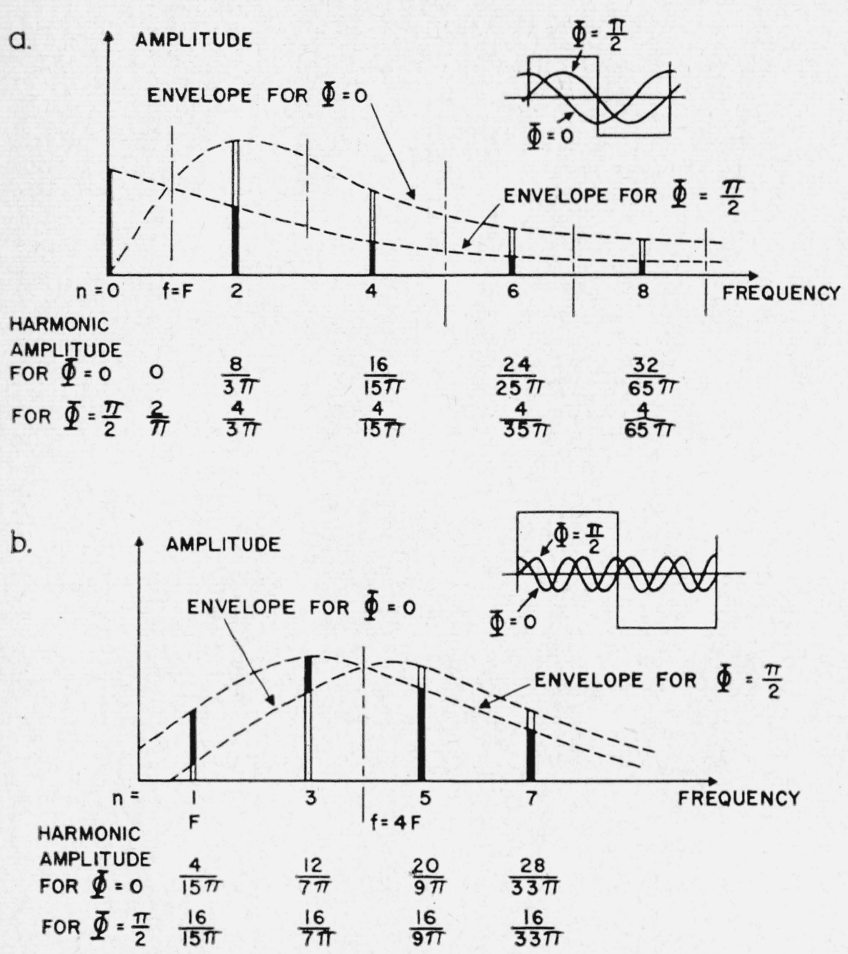

Figure 3. a, Harmonic spectrum for the case $m=1 ; b$, harmonic spectrum for the case $m=4$.

and its phase (eq 20):

$$
\phi_{n}=-\frac{n}{m} \tan \phi
$$

Figure 3 illustrates the harmonic spectrum in the two cases of $m=1$ and $m=4$. It is worthy of note that the amplitude of each harmonic is now dependent on the phase of the input, which is not true in the general case. This apparent contradiction may be explained if we think of the two families (a) and (b), which exist separately in the general case, as merging into one in the particular case when $f$ is a multiple of $F$ (the commensurable case). Depending on the relative phase of the two families (hence on the phase of the input, fig. 2) the resultant of each pair of merging harmonics will vary in amolitude.

In particular, note that the d-c component appears in the case of $m$ odd except when the phase of the input is zero or a multiple of $\pi$ (that is, when the input goes through a maximum, or minimum at each reversal of polarity). This may be verified by making $n=0$ and $\sin \phi=0$ in eq. $30 ; V_{n}$ vanishes under these conditions.

\section{Correlation between Time Constant and Selectivity}

One feature of the synchronous detector is of particular importance; its rejection of nonsynchronous input components. To achieve this, however,

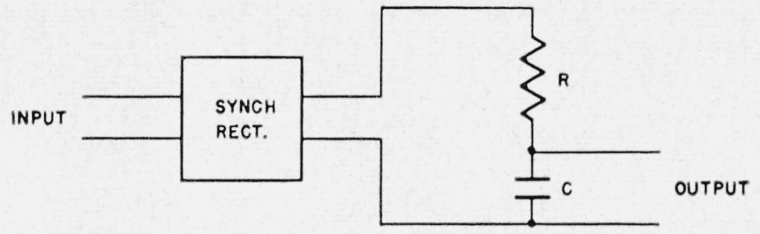

FIGURE 4. Output circuit of synchronous rectifier.

it becomes necessary to eliminate the a-c output of the synchronous detector. The foregoing analysis shows, in fact, that if the input contains, for example, the frequency $F+\Delta F$, slightly higher than the synchronous frequency, the output will contain all the frequencies $F+\Delta F \pm N F$ and $-f-\Delta F \pm N F$, and in particular, the frequency $\Delta F$, which, being low, cannot be eliminated without a network which will also delay amplitude changes in the synchronous signal.

In order to study this situation quantitatively, let us assume that the input frequency is capable of varying within a range and let us ask what time constant will be required, if we use a synchronous rectifier in conjunction with a resistance-capacitance (R-C) network, to achieve the same degree of selectivity as would be obtained with an ordinary rectifier preceded by a band-pass filter or the equivalent of selectivity $Q_{0}$. What we are looking for specifically is a correlation between $\tau$, the time constant of the R-C network, and $Q_{0}$, the selectivity ${ }^{4}$ of the equivalent band-pass filter. Suppose the input signal, of amplitude $V_{i}$, changes from the frequency $F$ to $F+\Delta F$. Assume, on the one hand, a band-pass filter such that this frequency deviation is accompanied by a reduction in half of the input energy. This filter will have selectivity of value:

$$
Q_{0}=\frac{F}{2 \Delta F} \cdot
$$

On the other hand, consider the synchronous rectifier. Before the shift this produced a d-c output of amplitude:

$$
V_{0}=\frac{2 V_{i}}{\pi}
$$

After the shift there will no longer be a d-c component, but there will be a component of frequency $\Delta F$ (the harmonic of family (b), fig. 2 , for $N=-1$ ). This will have amplitude:

$$
V_{0}=\frac{2 V_{i}}{2 \pi}
$$

Now suppose the synchronous rectifier is connected to the circuit of figure 4 . The change in peak voltage across the condenser due to the frequency shift will be due entirely to the discriminating action of the $\mathrm{R}-\mathrm{C}$ circuit, because aside from this the d-c and a-c peak values, as given by eq. 34 and 35 , are the same.

4 Selgin, Electrical transmission in steady state, pp. 180 and 295 (McGraw Hill Book Co., Inc. New York, N. Y., 1946). 
The peak voltage will therefore change in the ratio:

$$
r=\frac{1}{\sqrt{1+4 \pi^{2} \Delta f^{2} \tau^{2}}}
$$

where $\tau$ is the time constant of the R-C circuit. We are assuming that the condenser is not shunted by an appreciable load.

If we require the synchronous rectifier, with its $\mathrm{R}-\mathrm{C}$ circuit, to be as selective as the band-pass filter previously considered, the above ratio must be equal to $1 / \sqrt{2}$, resulting in the equation:

$$
4 \pi^{2} \Delta f^{2} \tau^{2}=1
$$

which, combined with (33), gives

$$
Q_{0}=\pi F \tau
$$

or, expressing the period $T$ of the synchronous frequency:

$$
Q_{0}=\pi \frac{\tau}{T}
$$

We therefore achieve, with the synchronous rectifier, an equivalent $Q_{0}$ equal to the ratio between the time constant and the period, multuplied by $\pi$. This result may serve to correlate selectivity with what we might call the "damping" of the synchronous rectifier: if instead of changing frequency we were to suddenly drop the amplitude of the input, the capacitor voltage (fig. 4) would fall exponentially, and the decrement (neglecting the source impedance of the input circuit which we assume to be small) would be

$$
\delta=\frac{I}{\tau}=\frac{\pi}{Q_{0} T}
$$

It is significant to compare with this the decrement associated with the build-up or decay of energy within the resonant circuit of selectivity $Q_{0}$ which, coupled to a nonsynchronous rectifier, was considered as an equivalent device. This second value of decrement may be derived from the equation

$$
Q_{0}=\frac{1}{2} \frac{\omega_{0}}{\alpha_{0}},
$$

which relates selectivity to the real and imaginary parts of the oscillation constant (decrement and angular frequency of the free oscillation). (See footnote 4). The decrement $\alpha_{0}$ is therefore given, identically, by

$$
\alpha_{0}=\frac{\pi}{Q_{0} T}
$$

What this means in practice is that the length of time required before the system reaches a new equilibrium after a change in input amplitude is the same whether we use a synchronous rectifier with $\mathrm{R}-\mathrm{C}$ network filtering or a nonsynchronous rectifier with a selective band-pass filter.

The gain in selectivity made possible by the use of synchronous rectifiers is largely due to the fact that very selective stable filters are difficult to realize, particularly at very low frequencies.

Washington, September 20, 1950 